\title{
Developing natural resource models using the object modeling system: feasibility and challenges
}

\author{
L. R. Ahuja ${ }^{1}$, J. C. Ascough II $^{1}$, and O. David ${ }^{2}$ \\ ${ }^{1}$ USDA-ARS-NPA, GPSRU, 2150 Centre Ave., Bldg. D, Suite 200, Fort Collins, CO 80526, USA \\ ${ }^{2}$ Colorado State University, Dept. of Civil Engineering, Fort Collins, CO 80523, USA
}

Received: 1 August 2004 - Revised: 1 November 2004 - Accepted: 15 November 2004 - Published: 9 August 2005

\begin{abstract}
Current challenges in natural resource management have created demand for integrated, flexible, and easily parameterized hydrologic models. Most of these monolithic models are not modular, thus modifications (e.g., changes in process representation) require considerable time, effort, and expense. In this paper, the feasibility and challenges of using the Object Modeling System (OMS) for natural resource model development will be explored. The OMS is a Javabased modeling framework that facilitates simulation model development, evaluation, and deployment. In general, the OMS consists of a library of science, control, and database modules and a means to assemble the selected modules into an application-specific modeling package. The framework is supported by data dictionary, data retrieval, GIS, graphical visualization, and statistical analysis utility modules. Specific features of the OMS that will be discussed include: 1) how to reduce duplication of effort in natural resource modeling; 2) how to make natural resource models easier to build, apply, and evaluate; 3) how to facilitate long-term maintainability of existing and new natural resource models; and 4) how to improve the quality of natural resource model code and ensure credibility of model implementations. Examples of integrating a simple water balance model and a large monolithic model into the OMS will be presented.
\end{abstract}

\section{Introduction}

The problems facing both developers and users of natural resource models are becoming increasingly complex. Tremendous progress has been made in discovering basic principles in different scientific disciplines that created major advances in management and technology for natural resource systems. However, understanding natural resource management issues related to ecology (habitat restoration), hydrology (water management), and farming practices (fertil-

Correspondence to: J. C. Ascough II

(jim.ascough@ars.usda.gov) izer and chemical application) become compounded when viewed within the physical, biological, chemical, and geological responses of the natural world. Computer simulations for prediction and management of watersheds, water supply areas, and agricultural fields and farms have also increased in complexity. The multidisciplinary nature of these problems usually requires accounting for a significant number of different models, data sources, management alternatives, and customers/stakeholders. This is particularly true in the case of agriculture where awareness of the general public requires careful management of agricultural systems in order to protect soil, water, and air quality. Simultaneously, market-based global competition in agricultural goods is challenging the economic feasibility of traditional agricultural production systems, and compels development of new and dynamic cropping and management strategies.

It can be argued that achieving the goal of sustainable natural resource management should involve consideration of whole system effects. Unfortunately, most natural resource systems involve highly complex interactions of soil-plantweather-management components that are extremely difficult to quantitatively describe. Thus, state of the art challenges in optimal management of the natural resources have created demand for integrated, flexible and easy to use modeling tools which are able to simulate the quantitative and qualitative aspects of the system (e.g., the hydrologic cycle) with a sufficient degree of certainty. Although a myriad of models are available, they are typically constrained to the specific scales and purposes they have been developed for and therefore are more robust in some areas than others (depending on the primary goal guiding their development). Furthermore, most of these monolithic models are not modular; are very difficult to update, add to, or connect with other models; have diminishing technical support; and lack the flexibility to meet current needs for more integrated analysis of changing natural resource issues.

All of the above reasons indicate a need for a new framework of model development that can integrate existing and future natural resource models into a common, collaborative, 
and flexible system. Such a system will maintain modularity, reusability, and interoperabililty or compatibility of both science and auxiliary components. The system will also recognize the fact that different categories of applications may require different levels of scientific detail and comprehensiveness, as driven by problem objectives, scale of application, and data constraints. These functionalities of the system will be obtained by establishing standard libraries of interoperable science and auxiliary components or modules that provide the building blocks for a number of similar applications. Module libraries have been successfully used in several domains, such as the manufacturing, transport, and other systems (Breunese et al., 1998; Praehofer, 1996). One of the earliest modular model developments was done for SHE, the European Hydrologic System Model (Abbot et al., 1986). Leavesley et al. (1996) reported the conversion of the Precipitation Runoff Modeling System (PRMS) to a Unix-based Modular Modeling System (MMS) for hydrologic modeling. Leavesley et al. (2002) presented some successful applications of this concept. Other examples of model integration framework initiatives include the Interactive Component Modelling System (Rahman et al., 2004), Tarsier (Watson and Rahman, 2004), Spatial Modeling Environment (Maxwell and Costanza, 1995, 1997), and HarmonIT (Blind and Gregersen, 2004). To summarize, an approach for modeling natural resource systems is needed that will:

- Reduce duplication of development effort, and improve the quality and currency of model code;

- Make natural resource models much easier to build, access, understand, and use;

- Facilitate long-term maintainability of existing and new natural resource models;

- Lead to greater consistency of modeling for particular problems and scales;

- Improve response and delivery times in scientific modeling projects;

- Ensure creditability and security of model implementations; and

- Function on any major computing platform.

The Object Modeling System (OMS) being developed by the USDA-ARS Great Plains Systems Research Unit (Fort Collins, CO) and the USGS (Denver, CO) meets the above criteria. The OMS provides a modular modeling framework which allows the implementation of single- or multi-process modules which can be compiled and applied as customtailored model assemblies.

\section{Object Modeling System (OMS) Description}

\subsection{Overview}

The OMS project was initiated in 1996 at the Friedrich Schiller University of Jena. In October 2000, the OMS evolved into an interagency project between the USDA-ARS, USGS, and USDANRCS, with financial support from ARS and inkind support from the partners. During the past 36 months, the OMS programming team has completed the development of most of the core components of the OMS. The OMS vision, described below for initial ARS implementation, is close to being realized (Ahuja et al., 2002):

"The OMS is a computer framework consisting of: 1) a library of science, control, and database modules; 2) a means to assemble the selected modules into a modeling package customized to the problem, data constraints, and scale of application; 3) automatic generation of a userfriendly interface; and 4) creation of a compiled, ready-to-run, version of the package. The framework is supported by utility modules such as data dictionary, data retrieval, GIS, graphical visualization, and statistical analysis. The framework employs the latest Java-based software technology for all its components. The science modules are also quickly updated or replaced as new knowledge and data become available. The OMS will be supported from a central server for use by all ARS scientists, NRCS specialists, USGS, and other collaborators."

The OMS is built on top of the NetBeans platform. The NetBeans platform is a framework for building desktop application software in the Java programming language. The OMS leverages NetBeans features such as user interaction components (e.g., menu bars, tool bars, status displays, tabbed-window displays, etc.), storage access components, and help components (e.g., JavaHelp). A schematic of OMS implementation for natural resource modeling is presented in Fig. 1.

\subsection{Component Architecture}

The general objectives of the OMS project included the development of generic software tools to extract modules from existing non-modular simulation models, and to incorporate them into the OMS framework with standard OMS descriptions. These tools have been developed, but need further testing and improvement. The OMS framework has the following functional components that are currently operational:

1. A module-building component that facilitates the integration of existing (legacy) code into the framework.

2. A module repository containing modules that can be readily utilized to assemble a working model (types of modules in the library will include science, control, utility, assessment, data access, and system modules).

3. A model builder that assembles modules from the module library into executable models and verifies data connectivity, and compatibility in scale and comprehensiveness. 


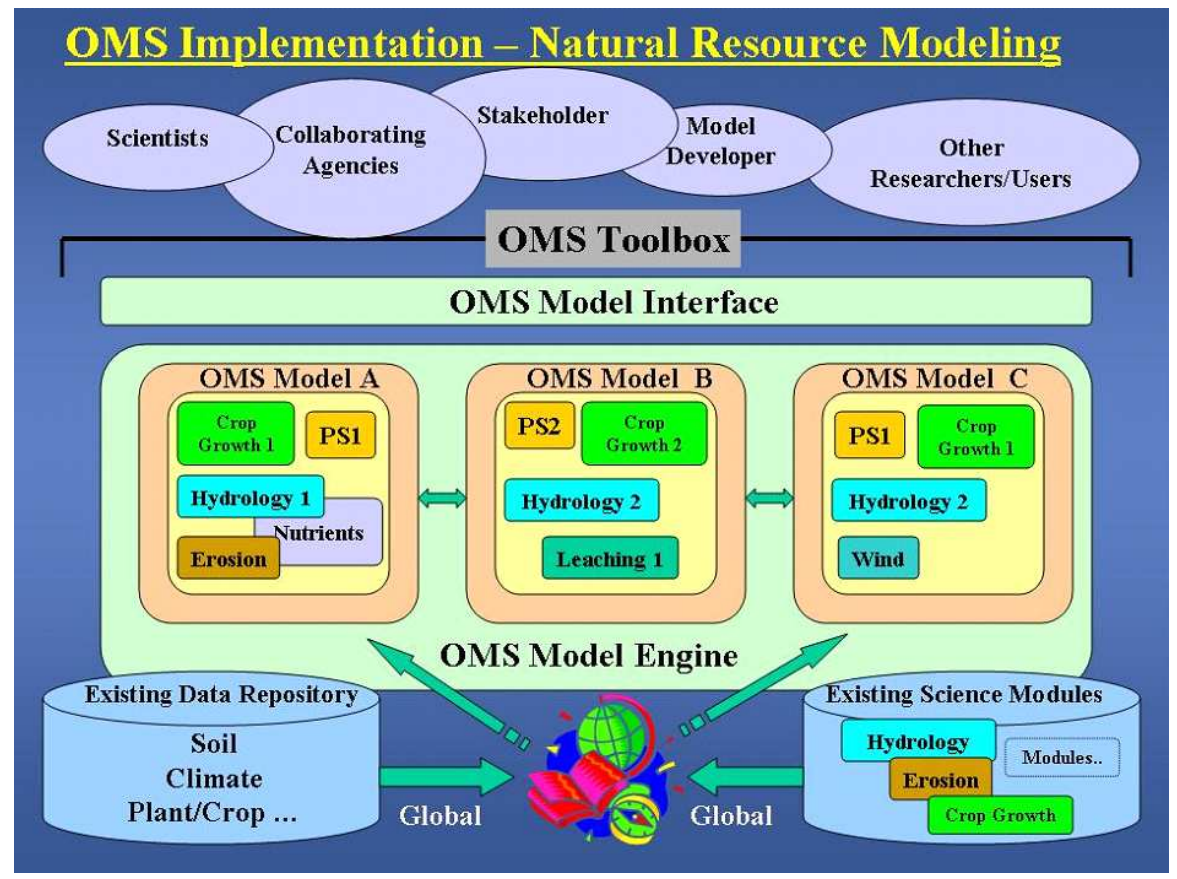

Fig. 1. Schematic of OMS implementation for natural resource modeling.

4. A dictionary framework that manages extended modeling data type information and provides extended semantics checking for module connectivity verification.

5. An extensible user interface that facilitates an appropriate user interaction for general model development and application (it is supported by a number of contributing software packages for database management, visualization, and model deployment).

The components have the following architecture or characteristics:

1. OMS models are treated as hierarchical assembled components representing building blocks. Components are independent and reusable software units implementing processing objects for simulation models. They reside in a model library and are categorized into data access components, science components, control components, utility components, and system components.

2. The OMS is able to integrate legacy code components. By an automated JAVA wrapper generation for legacy code, components written in languages such as Fortran or $\mathrm{C}++$ can be embedded into the OMS at the function level.

3. The "knowledge-backbone" of the OMS is the dictionary framework. It enables the OMS to verify state variables and parameters according to scientific nomenclatures during model development and application. Dictionaries are also used to specify parameter sets, model control information and the component connectivity.
They are implemented in the Extensible Markup Language (XML).

4. The OMS is extensible, i.e., extension packages exist for different aspects in model development and application. Extension packages are used for visual model assembly, model application, an interface to the dictionary framework, output visualization, and GIS integration.

5. The OMS scales from a full-featured, standalone development system with tools for model assembly, visualization, and analysis to a runtime Web service environment.

For a more complete explanation of the OMS framework and architecture, the reader is referred to David et al. (2002).

\section{Developing natural resource models with the OMS: advantages and disadvantages}

The following section lists the advantages (Ahuja et al., 2002) and disadvantages of developing natural resource models with the OMS.

\subsection{Advantages and feasibility}

1. Efficient transfer of technology: For the transfer of natural resource technology tools to stakeholders, researchers, and other users, the OMS will serve as a multidimensional platform for integration of various software tools. The end users will then develop deployment links to only the OMS, rather than develop links to each 
of the separate software tools as is current practice. This will result in a faster transfer of appropriate technology. For example, the USDA-NRCS Information Technology Center (ITC) in Fort Collins CO, USA has been a partner in OMS development, and is committed to using the OMS as a means to provide technical tools to 2500 field offices for natural resource conservation planning. The NRCS-ITC has already developed links to the OMS for its automated runoff curve number approach. Finally, due to a common model building platform and a common user interface for all models, the OMS will result in reduced start-up time for model development and lower training costs for users.

2. Cost reduction in maintenance and customer support for software technology: It is generally agreed that over the long-term, these items cost up to three times as much as for initial development of the software packages. At present, there are hundreds, if not thousands, of small to large natural resource software programs that need to be maintained and supported. A huge amount of time and labor is being spent on this process since monolithic models are becoming very expensive to use, difficult to update, have diminishing technical support, and lack the flexibility to meet todays needs for more integrated analysis of natural resource issues. These problems can be overcome if many of the existing packages were transferred to the OMS and all new packages were developed within the OMS. By using the existing Modular Modeling System (MMS) (Leavesley et al., 1996) for PRMS (Leavesley et al., 1983) model development and deployment, the USGS has realized a huge cost savings in maintenance and customer support. The web site http://iscmem.org/Memorandum.htm) lists details of a MOU that eight U.S. federal agencies (NRC, EPA, DOE, COE, USGS, ARS, NRCS, NOAA) have endorsed for the development and deployment of common methods and techniques for platform integration across all agencies.

3. Cost reduction in developing new software technology: In the past, model development efforts have primarily consisted of large teams of scientists. For example, ARS has had a number of individuals and teams build software technology and simulation models, including the erosion models WEPP and WEPS and the water quality models GLEAMS, RZWQM, AnnAGNPS, and SWAT. Each of these packages cost many millions of dollars to develop, including scientist and support time. Development costs were also inflated by significant duplication of work. Natural resource model developers can now leverage that investment by putting the science in those packages as modules in the OMS to build new customized software packages at a small fraction of the cost. Preliminary results in the core OMS development phase on modularization of the existing hydrologic models RZWQM and PRMS showed a code reduction of $20-33 \%$ for OMS model versions while preserving the same simulation results. In this age of information technology, the demand for such software packages will increase tremendously. In the ARS alone, if we were to develop ten customized large new system packages to meet this demand over the next ten years, we will save at least $\$ 100$ million dollars, assuming $80 \%$ of the science comes from existing modules already put in the OMS and $20 \%$ is new code.

4. Applying the most suitable science for specific problems: The OMS will allow the selection of the best evaluated and most appropriate science modules currently available depending upon the nature of the problem and required answers, availability of input data, and scale of application. The OMS library may have different modules for a research model versus a management decision tool. Similarly, a watershed-scale management model may possibly require different (i.e., less complex) science modules than a field-scale model. Issues of dimensionality (e.g., 1-D surface vs. 2-D groundwater), scale, or dynamic interactions (e.g., feedback mechanisms) between modules can be easily handled in the OMS as long as the required module structures and data dependencies are adequately defined.

5. Assure reliability in results from software tools for similar applications: Natural resource software application users often report that different software tools or simulation models give vastly different results, say for predicting crop yield, because the tools used different science approaches in key process areas. The OMS will significantly reduce this problem by utilizing evaluated, documented, and standardized modules for the basic science components for a given category of applications.

6. The OMS library as a reference and coordination tool for future research and development: The OMS library will be a repository of current, quantitative knowledge in different areas of natural resource system science. Future scientists could look to this library to help determine where further research and development are needed.

7. Integrated analysis of natural resource system production and conservation issues: Effective analysis and management of natural resource systems and the environment requires integration of tools and data types that now exist in an array of individual disparate models. The OMS will provide customized, whole-system tools for the analysis of production and conservation issues (e.g., environmental quality and global climate change management) in natural resource systems.

8. OMS certification mechanism for approved "science building blocks": The OMS supports the technical certification of library components based on X.509 Certificates and the validation of such certificates. This will 


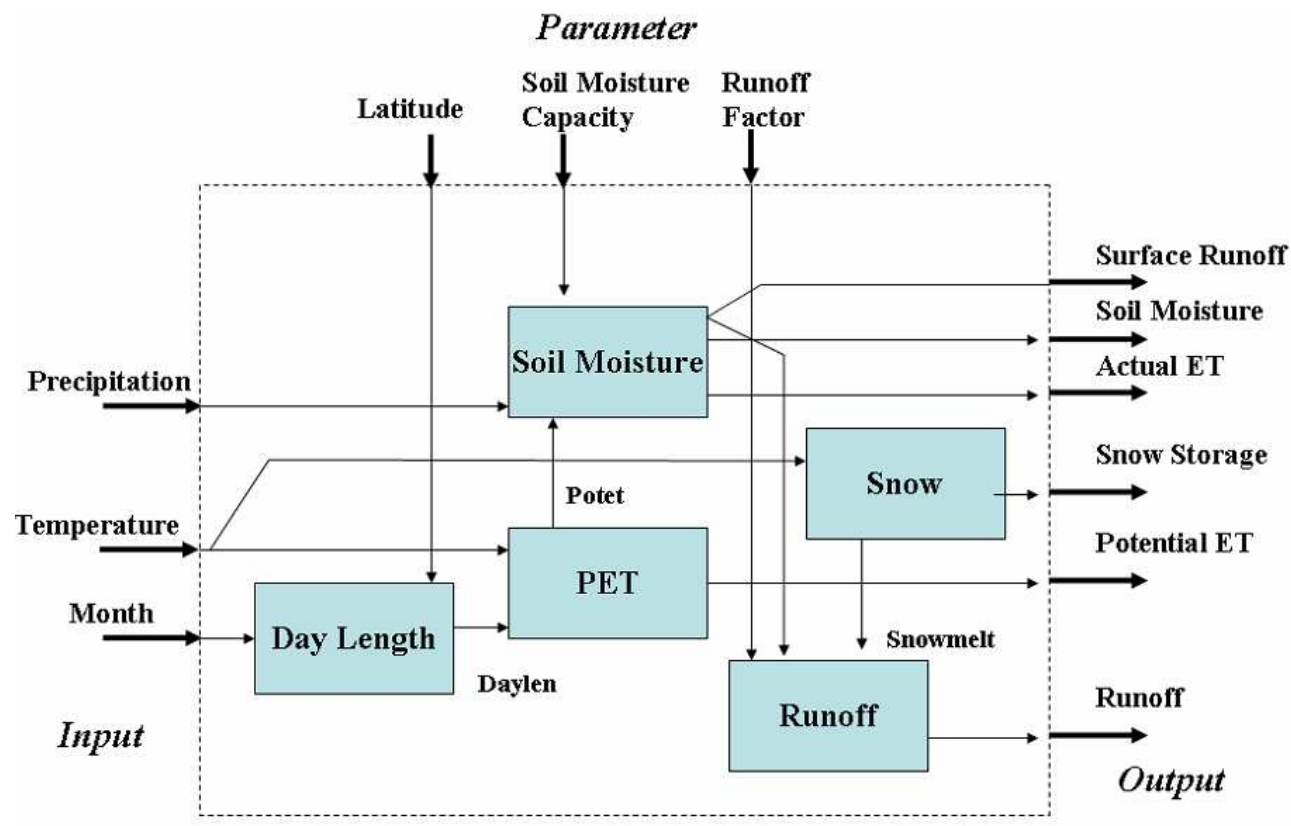

Thornthwaite Water Balance Model

Fig. 2. OMS components and Thorthwaite water balance model assembly.

allow agencies or other entities to certify approved modeling components and models. In addition, license protection of modules or components is allowed through signatures within the Netbeans platform.

9. Enhance productivity of scientists and researchers: The customized, best-quality, software tools developed through the OMS will help field scientists quantify their results and transfer them to other soils and climates very rapidly. The gaps identified in the process will make future research more focused. Overall, the productivity of scientists and the quality of science should increase as focus centers on science module implementation rather than Graphical User Interface (GUI) design, software deployment, packaging and maintenance.

10. International coordination in new science module development and publication: Through the Internet, the OMS will serve as a common platform for international scientists and researchers to contribute their findings as modules to the OMS library. A supervisory group or organization (e.g., International Association of Hydrological Sciences) could coordinate this development, and provide a mechanism for peer review and quality control. The module contribution to the library will be considered a publication by scientists that could have world-wide impact.

\subsection{Challenges and difficulties}

Challenges to using the OMS for natural resource model development stem from the following problems:
- Lack of motivation to share model code - in order to fulfill the intended purpose of the OMS, model developers must build a repository of modules through contribution to the OMS module library.

- Acceptance of a modular coding structure - model developers must spend more "up-front" time in module development in terms of module structure, $\mathrm{I} / \mathrm{O}$ requirements, metadata description, etc.

- Willingness to share data sets for a range of natural resource processes covering different climatic and physiographic regions across the world - application of natural resource models developed under the OMS will be difficult without data sets for comparison and evaluation.

- Loss of model name recognition - this can be overcome, however, by the development of a mechanism for peer review and quality control of individual modules.

\section{Results and conclusions}

4.1 OMS integration example: Thornthwaite water balance model

The OMS utilizes Component Oriented Programming (COP) for the creation and execution of simulation models. Components in general take the idea of object-orientation to a next level. While object-oriented design methods support abstraction and localization of data and methods, they can also lead 


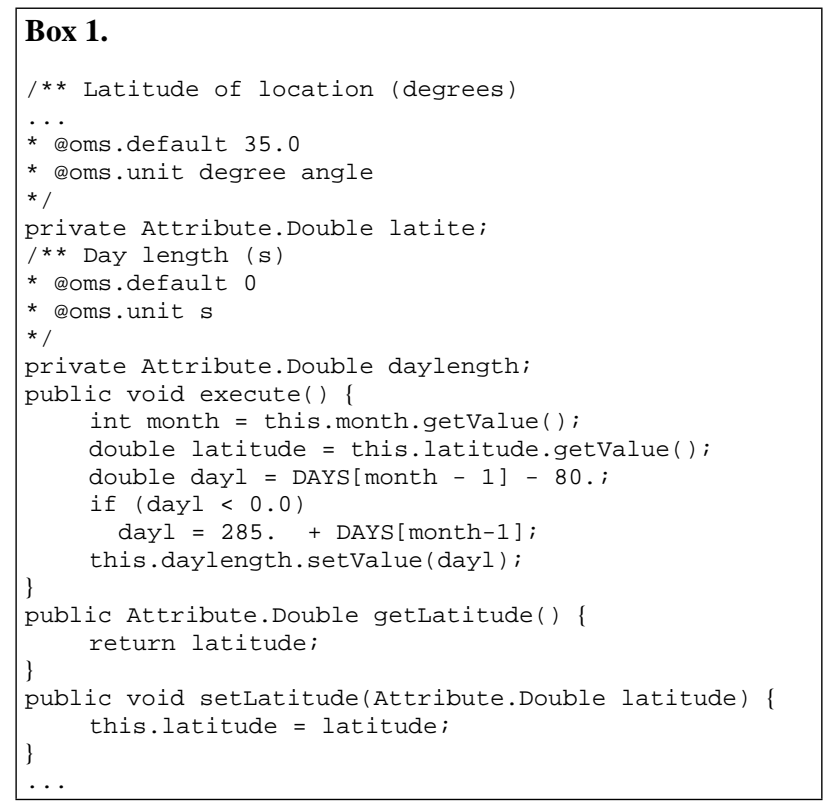

to simulation systems where objects are codependent. To remove that limitation, a more rigid idea was introduced in the OMS: the component. The key difference between a standard object and a component in the OMS is that a component is completely replaceable and provides its behavior by having a passive structure. This means that the simulation component is always called from the system, as opposed to the component calling some system functionality. This approach provides the highest degree of flexibility from a modeling framework point of view.

This section presents an example of an OMS natural resource model comprised of small, well-defined components. The Thornthwaite water balance model (Thornthwaite, 1948; Mather, 1978, 1979) uses an accounting procedure to analyze the allocation of water among various components of the hydrologic system. Inputs to the model are monthly temperature $\left({ }^{\circ} \mathrm{C}\right)$ and precipitation $(\mathrm{mm})$. Outputs include monthly potential and actual evapotranspiration, soil moisture storage, snow storage, surplus, and runoff (all in $\mathrm{mm}$ ). The connectional structure is shown in Fig. 2. The model consists of five components for (1) computing day length, (2) potential evapotranspiration (PET), (3) soil moisture accounting, (4) snow accounting, and (5) runoff generation.

Each component represents a hydrological process concept and the inputs/outputs are well-defined. There is internal data flow between the components, as well as external available Input and Output slots (Fig. 2). Box 1 shows a code fragment of the Daylen component with the definition of the parameter "latitude" and the output variable "daylen". The "execute()" method implements the computation of the day length based on current month and latitude. The Daylen component provides accessor methods (e.g., setLatitude/getLatitude) allowing the OMS to pass data according to the metadata specifications above each data declaration.

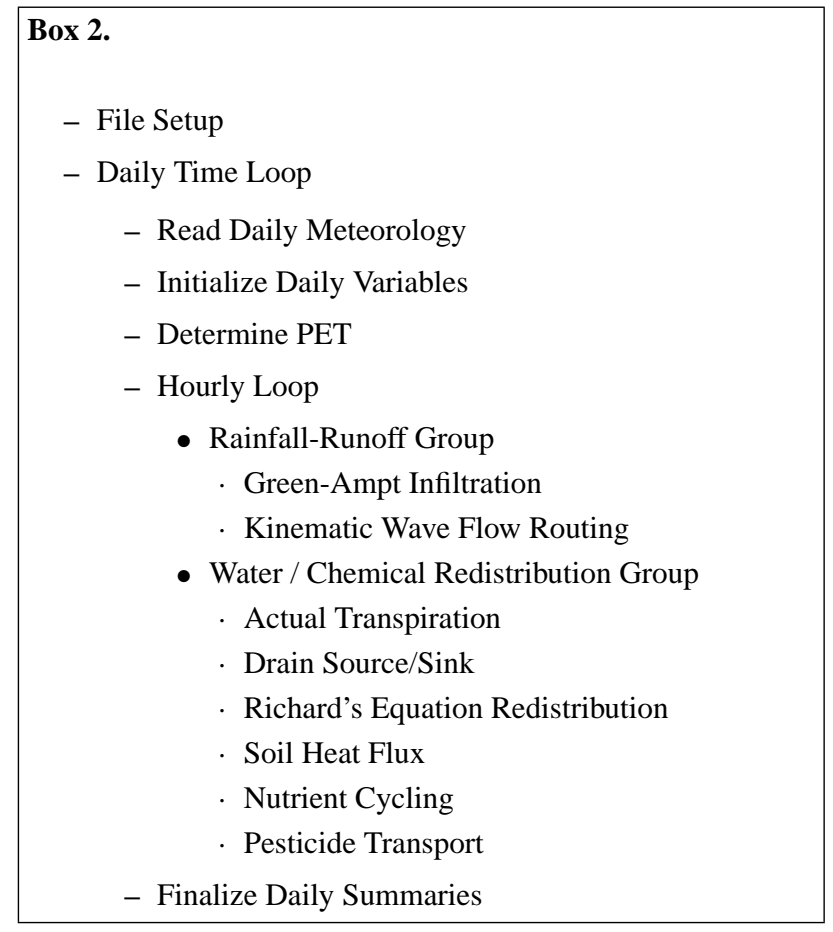

To run the Thorthwaite water balance model, the OMS connects the input with the system input reader and allows each variable to be plotted as output. The components reside in a "TimeContainer" which handles the monthly iterations based on the input data set.

\subsection{OMS Integration Example: RZWQM}

As previously stated, creating highly integrated natural resource simulation models using traditional programming methods can be expensive and time-consuming. This section presents an example of using the OMS to create a new agricultural water quality model utilizing component-oriented methods coupled with process-based modules. A large-scale Fortran 77 simulation model, the Root Zone Water Quality Model (RZWQM) (Ahuja, 2000) was disaggregated into smaller components which were then reassembled into a functionally identical model within the OMS. RZWQM integrates physical, biological, and chemical processes to simulate plant growth and the movement of water, nutrients, and pesticides over and through the root zone for a representative unit area of an agricultural field over multiple years. Box 2 shows a simple schematic of the organizational structure of the RZWQM model represented in the OMS to complete the simulations.

A challenge of monolithic model disaggregation is deciding at which point in the original code it is reasonable to extract a new section of code into a singular executable component. This extraction process is typically iterative with the intended result being a module containing a level of complexity that makes sense for the intended use. In other words, module size and complexity should be determined by functional use, e.g., modules consisting of single algorithms may 


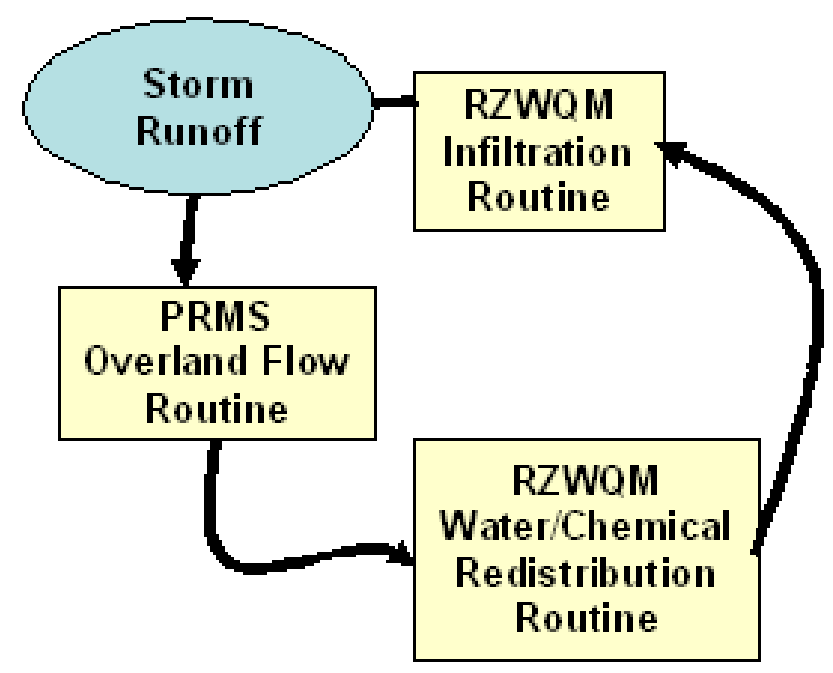

Fig. 3. RZWQM Green-Ampt infiltration routine generates runoff during a storm event and is passed to the PRMS overland flow routine to drive the kinematic wave solution (if no storm event, a Richards equation based redistribution routine is used).

be appropriate or an entire monolithic model may function as a module. The OMS-based version of RZWQM was then extended with components for runoff and soil erosion to form a new simulation tool to examine water quality assessment at a small watershed scale. Point estimates of rainfall excess (runoff) generated in RZWQM infiltration components were routed through the PRMS-based kinematic wave routine to move the water to the edge of an overland flow plane (Fig. 3). In the future, this runoff/runon water would be simulated for a series of overland flow planes that could then be routed through a channel routing routine to simulate watershed hydrology. Figure 4 shows the sample output hydrograph in the OMS from a test input rainfall event.

\subsection{Concluding Remarks}

A large number of complex natural resource system models are currently in use worldwide. Most of these models are based on sound science, but have specialized data requirements and significant duplication exists in many areas. The current OMS development tool will leverage these sizeable investments of developer time and money to: 1) facilitate an interdisciplinary effort extracting the best scientific routines of existing models; and 2) provide integration and interoperability of existing and new scientific modules and modern data resources. To summarize, principal advantages of the OMS include:

1. The OMS will increase the probability of using the best science available in various combinations for the given conditions and problem.

2. The OMS will be easier to maintain and update as new knowledge, data and technology become available. The OMS will allow a "select, plug, and play" mechanism

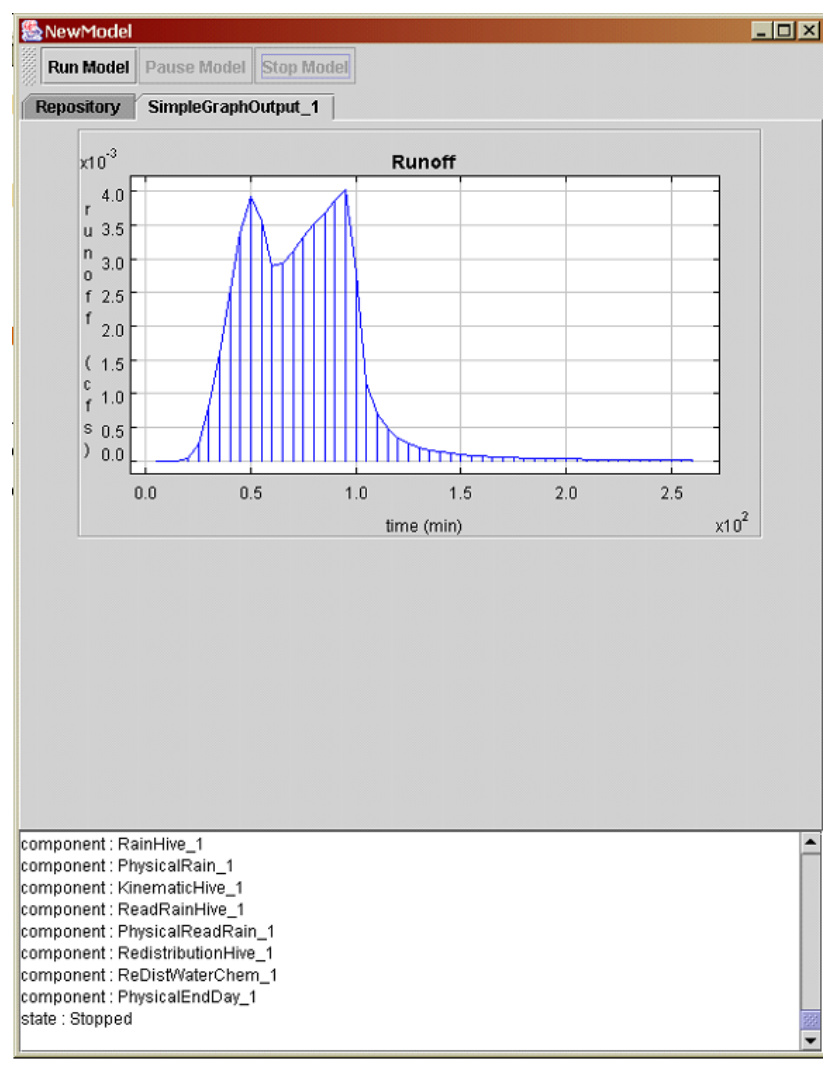

Fig. 4. Results from the OMS simulation show the runoff (cfs) hydrograph.

for modules consisting of sub-models, equations, graphics, statistics, risk analysis, parameter estimation, standard data sources and various reporting formats.

3. New knowledge expressed in the form of modules will be relatively easy to verify and evaluate (and possibly lead to scientific peer review).

4. The OMS will help to eliminate duplicate functionality across natural resource models. The OMS library of modules will serve as a reference and a coordination mechanism for future improvements. The OMS will facilitate communication between model developers by providing a common standard for development and implementation.

5. The OMS should significantly reduce the problem of different natural resource models giving different results by utilizing a library of evaluated, documented, and standardized modules and integrated output options.

6. The OMS will provide a consistent interface for model creation and evaluation, and will reduce startup time for scientific users and developers.

7. The OMS offers support-ready compliance with the distinct advantage of having a set of application packages (e.g., data input, parameterization, visualization) under 
a single user interface or a set of consistent user interfaces.

8. The OMS will allow flexibility to choose scientific modules most appropriate for the scale or region of interest, or to respond to other unique influencing factors under consideration.

In conclusion, the component-oriented and modular approach of the OMS and the modules/models implemented in it will provide the basis for more efficient and collaborative model development in the future. This type of integrative and open-source approach is desperately needed in order to solve global challenges impacting natural resource systems such as sustainable management of natural resource systems and the impact of global climate change on natural resource systems. For more details on the OMS project mission, project documentation, or to download the entire application or individual modules, visit the OMS web site at http://oms.ars.usda.gov/.

Acknowledgements. The authors wish to thank I. Schneider and K. Rojas for their programming support on OMS core model development.

Edited by: P. Krause, S. Kralisch, and W. Flügel

Reviewed by: anonymous referees

\section{References}

Ahuja, L. R., Rojas, K. W., Hanson, J. D., Shaffer, M. J., Ma, L. (Eds.): Root Zone Water Quality Model, Water Resources Publication, 2000.

Ahuja, L. R., David, O., Blackburn, W., Amerman, R., Werner, J., Carlson, J., Knighton, R., and Leavesley, G.: The Object Modeling System (OMS): an advanced object-oriented, modular modeling computer technology for agricultural production systems, USDA-ARS Working White Paper, Vers. 1, Fort Collins, CO, 2002.

Abbot, M. B., Bathurst, J. C., Cunge, J. A., O'Connell, P. E., and Rasmussen, J.: An introduction to the European Hydrological System-Systeme Hydrologique Euopeen, "SHE", 2: structure of a physically-based, distributed modelling system, J. Hydrol., 87, 61-77, 1986.

Blind, M. and Gregersen, J. B.: Towards an Open Modelling Interface (OpenMI) The HarmonIT project, in: Complexity and Integrated Resources Management, edited by: Pahl-Wostl, C., Schmidt, S., Rizzoli, A. E., and Jakeman, A. J., Transactions of the 2nd Biennial Meeting of the International Environmental Modelling and Software Society (iEMSs), Manno, Switzerland, ISBN 88-900787-1-5, 346-351, 2004.
Breunese, A. P. J., Top, J. L., Broenink, J. F., and Akkermans, J. M.: Libraries of reusable submodels: theory and application, Simulation, 77 (1), 7-22, 1998.

David, O., Markstrom, S. L., Rojas, K. W., Ahuja, L. R., and Schneider I. W.: The Object Modeling System, in: Agricultural System Models in Field Research and Technology Transfer, edited by: Ahuja, L. R., Ma, L., and Howell, T. A., Lewis Publishers, 317-330, 2002.

Leavesley, G. H., Lichty, R. W., Troutman, B. M., and Saindon, L. G.: Precipitation Runoff Modeling System: Users Manual, Water Resources Investigations 83-4238, USGS, Denver, Colorado, 1983.

Leavesley, G. H., Restrepo, P. J., Markstrom, S. L., Dixon, M., and Stannard, L. G.: The Modular Modeling System (MMS): Users Manual, Open File Report 96-151, USGS, Denver, Colorado, 1996.

Leavesley, G. H., Markstrom, S. L., Restrepo, P. J., and Viger, R. J.: A modular approach to addressing model design, scale and parameter estimation issues in distributed hydrological modelling, Hydrol. Proc., 16, 173-187, 2002.

Mather, J. R.: The Climatic Water Balance in Environmental Analysis, D.C. Heath and Company, Lexington, Massachusetts, 239 pp., 1978.

Mather, J. R.: Use of the climatic water budget to estimate streamflow, in: Use of the Climatic Water Budget in Selected Environmental Problems, edited by: Mather, J. R., Publications in Climatology, 32, 1-52, 1979.

Maxwell, T. and Costanza, R.: Distributed modular spatial ecosystem modelling, Int. J. Comp. Simul.: Special Issue on Advanced Simulation Methodologies, 5 (3), 247-262, 1995.

Maxwell, T. and Costanza, R.: A language for modular spatiotemporal simulation, Ecol. Modell., 103 (2,3), 105-114, 1997.

Praehofer, H.: Object oriented, modular hierarchical simulation modeling: towards reuse of simulation code, Simul. Pr. Theory, 4 (4), 221-234, 1996.

Rahman, J. M., Seaton, S. P., and Cuddy, S. M., Making frameworks more useable: using model introspection and metadata to develop model processing tools, Env. Modell. \& Soft., 19 (2004), 275-284, 2004.

Thornthwaite, C. W.: An approach toward a rational classification of climate, Geographical Review, 38, 55-94, 1948.

Watson, F. G. R and Rahman, J. M.: Tarsier: a practical software framework for model development, testing, and deployment, Env. Modell. \& Soft., 19 (2004), 245-260, 2004. 